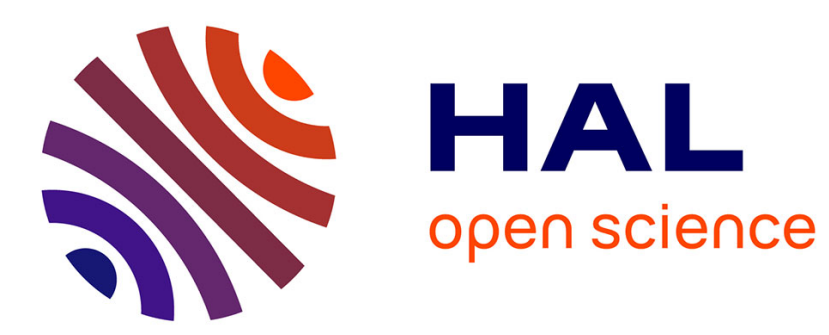

\title{
We Need Non-formal Methods Based on Formal Models in Interaction Design
}

Andreas Maier, Steffen Hess

\section{To cite this version:}

Andreas Maier, Steffen Hess. We Need Non-formal Methods Based on Formal Models in Interaction Design. 7th Workshop on Human-Computer Interaction and Visualization (HCIV), Aug 2011, Rostock, Germany. pp.150-164, 10.1007/978-3-642-54894-9_11 . hal-01414702

\section{HAL Id: hal-01414702 \\ https://hal.science/hal-01414702}

Submitted on 12 Dec 2016

HAL is a multi-disciplinary open access archive for the deposit and dissemination of scientific research documents, whether they are published or not. The documents may come from teaching and research institutions in France or abroad, or from public or private research centers.
L'archive ouverte pluridisciplinaire HAL, est destinée au dépôt et à la diffusion de documents scientifiques de niveau recherche, publiés ou non, émanant des établissements d'enseignement et de recherche français ou étrangers, des laboratoires publics ou privés. 


\title{
We need Non-Formal Methods based on Formal Models in Interaction Design
}

\author{
Andreas Maier and Steffen Hess \\ Fraunhofer Institute for Experimental Software Engineering IESE \\ \{andreas.maier, steffen.hess\}@iese.fraunhofer.de
}

\begin{abstract}
According to our experience, early collaboration with non-expert stakeholders aimed at designing interaction in a user-centered way is mandatory if the goal is a great user experience. We have found that insistence on formal modeling when collaborating with non-experts leads to insufficient results. Therefore, we propose a user-centered approach in order to enable collaboration and communication among expert and non-expert stakeholders. This nonformal approach should be based on a formal model, which also builds the common ground for discussions between all involved project stakeholders.
\end{abstract}

\section{Introduction}

\subsection{Motivation}

Well-designed and usable human-computer interaction (HCI) is a key factor for successful software products. Offering a large number of features is no longer sufficient for achieving success on the market. We observe stronger interest in usability and user experience factors in the decisions people make regarding particular products in the same class of devices (e.g. iPhone vs. other smartphones, iPod vs. other MP3 players). Users expect good usability and want to enjoy a great experience when interacting with the system. But what makes an interaction a great experience? What are the elements of a great HCI? Which dependencies exist between these elements? How do we design such an interaction? These are the questions we want to answer in order to improve the results of interaction design and contribute value to the HCI community. To do so, we developed a formal interaction model, which shows the most important elements of HCI and their dependencies. This model is called MAInEEAC (Model for Accurate Interaction Engineering, Enhancement, Alteration, and Characterization) and is introduced briefly in section 2 . Since we are aware of the fact that a model is not applicable to conversations between expert stakeholders and non-expert stakeholders, we also created and use the non-formal interaction design method "mConcAppt", which is built on MAInEEAC and tailors HCI elements to the given context in which an $\mathrm{HCI}$ is designed. During this article, we refer to experts as experts in terms of software development.

adfa, p. 1, 2011.

() Springer-Verlag Berlin Heidelberg 2011 
After the description of MAInEEAC, we describe our interaction design method in section 3. Section 4 sums up the advantages of our approach. The article closes with a conclusion and an outlook on future work.

\subsection{Our Model-Based Interaction Design Approach}

In contrast to purely formal model-based approaches or the strict performance of formal methods, we apply a user-centered design approach [6], which combines the advantages of both formal models and non-formal methods.

In this approach, we involve non-expert stakeholders as early as possible, since only potential operators of the system under development know what constitutes a great experience in their context. When non-expert stakeholders are involved, experts use formal models to work effectively and efficiently: Every expert has a formal conceptual model of the target system in mind when designing an interaction [7], in this case a formal model for interaction design. With the help of this model, the experts are aware of every element of $\mathrm{HCI}$ and every dependency between elements. In conversations with non-expert stakeholders, the experts are able to ask for all relevant information regarding $\mathrm{HCI}$, trace the development of the $\mathrm{HCI}$, and find conflicting specifications immediately by matching the conceptual model to the non-expert stakeholders' mental models of the system [ibid.]. To prevent non-expert stakeholders from being confused, the expert does not show the formal model to them, but only uses it as preparation and for post-processing.

With the formal model in mind, the experts can describe a particular HCI on a very detailed level. They can easily show interaction elements and dependencies, can facilitate communication between stakeholders involved in the development process, and close the gap between interaction designers and end users.

The non-formal method is used for eliciting, analyzing, and specifying requirements, prototyping $\mathrm{HCI}$ according to the elements given by the formal model, and validating the interaction design.

\section{MAInEEAC - A Formal Model for Interaction Engineering, Enhancement, Alteration, and Characterization}

As the underlying model of our user-centered interaction design approach, we developed MAInEEAC - Model for Accurate Interaction Engineering, Enhancement, Alteration, and Characterization [4]. This model is view-based and currently consists of ten views, six of which we present in detail in this report. These six views are most relevant for this approach and build the formal basis of the non-formal method. The four views we do not describe in this report represent even more details of HCI and are mainly interesting for researchers in this area, currently. They deal with characteristics of users, with the process of information perception and its details, respectively, and with different types of interactions. 


\subsection{General Overview}

Figure 1 gives an overview of the interaction flow as described in MAInEEAC. This basic and coarse overview conforms to the unanimous view on HCI taken by almost every area dealing with HCI. Triggered by an Intention, the Human initiates an action on the Input Interface of the system. The System processes the given input and delivers a system reaction via its Output Interface. The Human again perceives this as a reaction to his Intention and evaluates if the perception is appropriate. In any case, this perception might affect the subsequent Intentions. This short description shows the basic concept of our model. As mentioned above, this view is common sense in the field of HCI. The concepts follow the Gulf Model published by Donald Norman in 1988 [8]. The distinct feature of MAInEEAC is that it is not restricted to the general overview shown in Figure 1, but shows all aspects in detail as well. It enables the different roles involved in system development to work with only one model. MAInEEAC represents the system in great detail, without being system-centered. In fact, it is interaction-centered, emphasizing the human at the same time. Cognition is not an explicit element in MAInEEAC, but implicitly covered by Perception, which covers the recognition, interpretation, and evaluation of information. Media and Modalities are well-defined distinct elements of HCI, both with an exact meaning: Media are representation forms of information, while Modalities are the concrete usages of human senses to perceive information. The user interface and possible system outputs are decomposed in order to describe $\mathrm{HCI}$ in even greater detail. Overall, MAInEEAC enables us to describe an interaction with a system without having to use another model.

We decided on using a view-based representation of the model to emphasize certain aspects of HCI. The following sections describe the most important aspects of MAInEEAC, including detailed views on its basic components (human action, system, system action, and interaction).

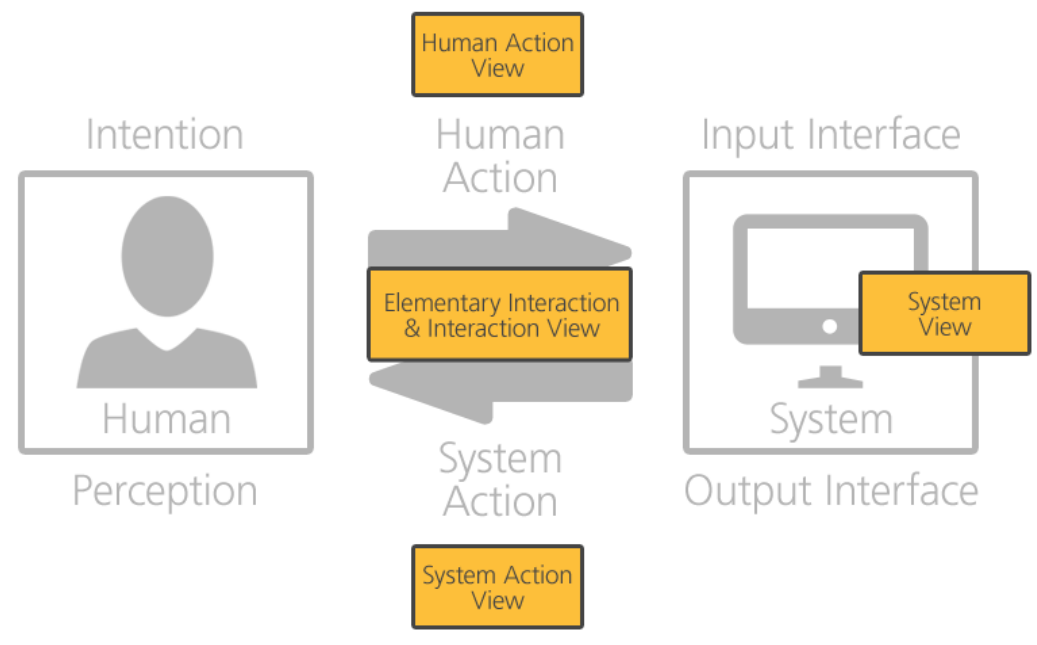

Fig. 1. MAInEEAC - General Overview 


\subsection{System View}

The System View describes the system side during HCI (see Figure 2). MAInEEAC does not treat the System as a black box like many other HCI models do [8],[9]. The System components highly influence HCI and thus are shown in the same detail as Human elements and elements of the Interaction itself.

In general, we define System as a complex object based on software that fulfills a function by processing input and creating output. Systems in the application area of MAINEEAC might be PCs, industrial machines, handhelds, home appliances, consumer electronics, etc.

Each System consists of a number of devices. A Device is a technical aid which acts as interface for transmitting information from human to system and / or from system to human. Furthermore, devices are all parts of the system, no matter if these parts are actually used for the interaction or not (e.g. an electric shutter being controlled with the system). A device acting as Input Interface transmits information from human to system (e.g., keyboard, PC-mouse, touch-screen, microphone, and digitizer), whereas a device acting as Output Interface transmits information from system to human (e.g., screen, loudspeaker, and braille-display).

A System communicates with its environment through its User Interface, which presents the aggregation of all Input Interfaces and Output Interfaces.

The important fact we show with this distinction between device, input interface and output interface is that input and output might take place on different devices and even at different locations during HCI.

Every Input Interface is characterized by the Usage Types it offers. A Usage Type specifies how an Input Interface is used concretely to transmit information to a system (see also section 2.2 for a further clarification).

Furthermore, an Input Interface may give Direct Input Feedback which comes straight from the Device without the use of any Medium (for example, the sound that occurs when pressing a button on a mouse or keyboard). When a Device acts as an Output Interface, it gives Application Feedback and might give Indirect Input Feedback in addition. Both are transmitted via a Medium: Indirect Input Feedback is a reaction to the usage of the Input Interface to confirm the system's correct understanding of the human's action, for example, highlighting of a selected menu item transmitted via the Medium "graphic". It is always based on a System Function. The complete decomposition of system actions and detailed descriptions of each possible system action is represented in section 2.4. 


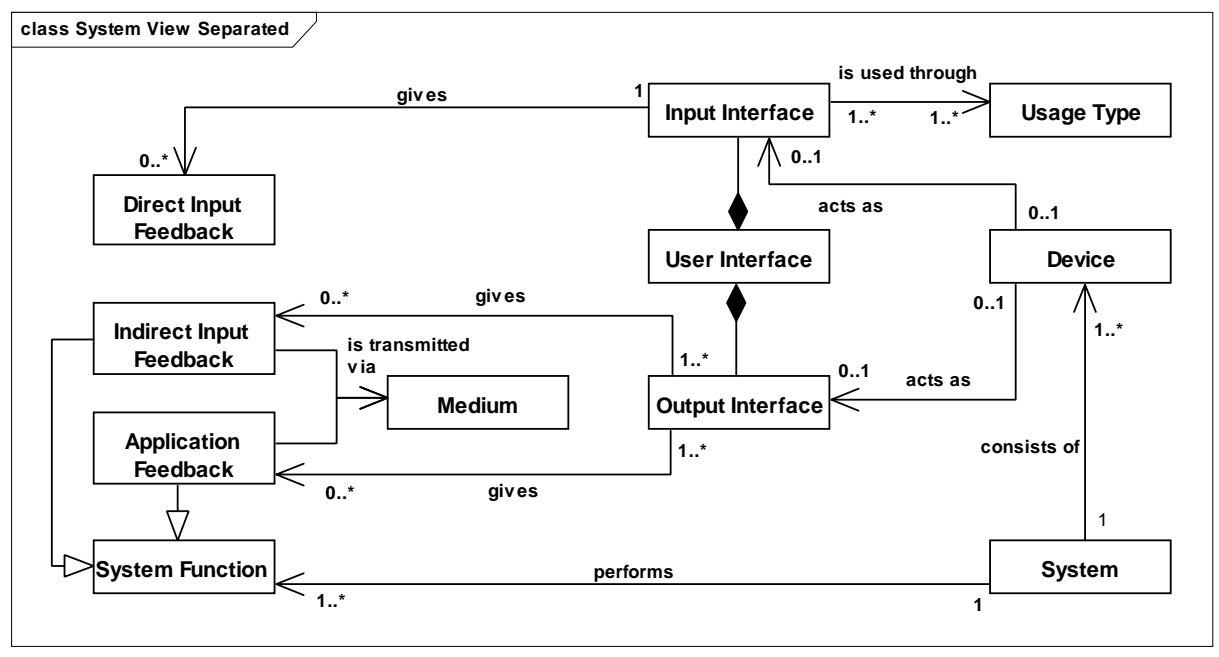

Fig. 2. System View

\subsection{Human Action View}

The Human Action view (see Figure 3) describes the way the Human accomplishes his Intention on the Input Interface of the System. In MAInEEAC, this accomplishment is called Human Action and involves the whole activity performed by a Human to transmit information to a System. Every activity might be influenced by the Environmental Context in which the activity takes place. For example, the Human might not want to transmit information to the System via speech when he is in a noisy environment or he might want to interact with the System from a distance when his environment is a huge warehouse. The Environmental Context is an attribute of Human Action and thus has to be specified when using MAInEEAC. Each Human Action consists of at least one Action Method, which specifies the action of the Human. It comprises generic human movements according to original human abilities. We distinguish four types of action methods:

1. Fine Motor Skills (e.g., typing, writing, moving an object, pressing an object)

2. Gross Motor Skills (e.g., gesturing, walking, jumping)

3. Facial Expressions (e.g., smiling, grinning, frowning)

4. Vocal Utterances (e.g., speaking, whispering, shouting)

Furthermore, a Human Action always has a Method Type, which specifies if the action is uni-methodical or multi-methodical. If there is only one kind of Action Methods, the type is uni-methodical. If there are at least two Action Methods, the Method Type is multi-methodical.

When performing the Human Action, every Input Interface features different Usage Types that specify how an Input Interface is exactly used to transmit information to the System. For example, 'pressing object' as an Action Method is underspecified. There is a set of concrete movements being performed within a Human Action. It 
makes a big difference whether one presses the left or the right mouse button or whether someone performs a single or a double click. In addition, the Usage Type concretely specifies the Action Method. For example, if the Usage Type of the Input Interface is 'single left click', the Action Method is determined as 'pressing object'.

When characterizing an interaction with MAInEAAC, the elements Action Method, Usage Type, and Input Interface influence each other. When an Action Method is determined, the possible Input Interfaces and Usage Types are deduced from that Action Method. If, for instance, the Action Method 'Speaking' is determined, the number of Input Interfaces possible for sound input is low and the possible Usage Types are restricted to that Action Method. The Action Method in conjunction with the Usage Type restricts the number of possible Input Interfaces even more. With respect to the Usage Type, the same holds for the Action Method in conjunction with the Input Interface.

The Usage Type determines the Action Method and restricts the number of available Input Interfaces to those that offer the specified Usage Type and allow for performing the deduced Action Method. For example, when the Usage Type 'Natural Speech' is selected, 'Speaking' is automatically determined as the Action Method. The list of Input Interfaces is restricted to different microphones like desktop microphone, handheld microphone, wearable microphone, etc. When we decide on a particular Input Interface, the Action Method as well as the Usage Type are restricted at the same time. When Input Interface and Usage Type have been determined, the Action Method is deduced from those. This does not hold for the determination of the Input Interface and the Action Method: When the Input Interface and the Action Method have been determined, we still have a choice regarding the Usage Type. For example, when we decide on 'microphone' as Input Interface and 'natural speech' as Usage Type, 'Speaking' is automatically determined as the Action Method. When we leave the Usage Type open and decide on an Action Method from the class 'Vocal Utterance', we can still decide on which Usage Type to apply.

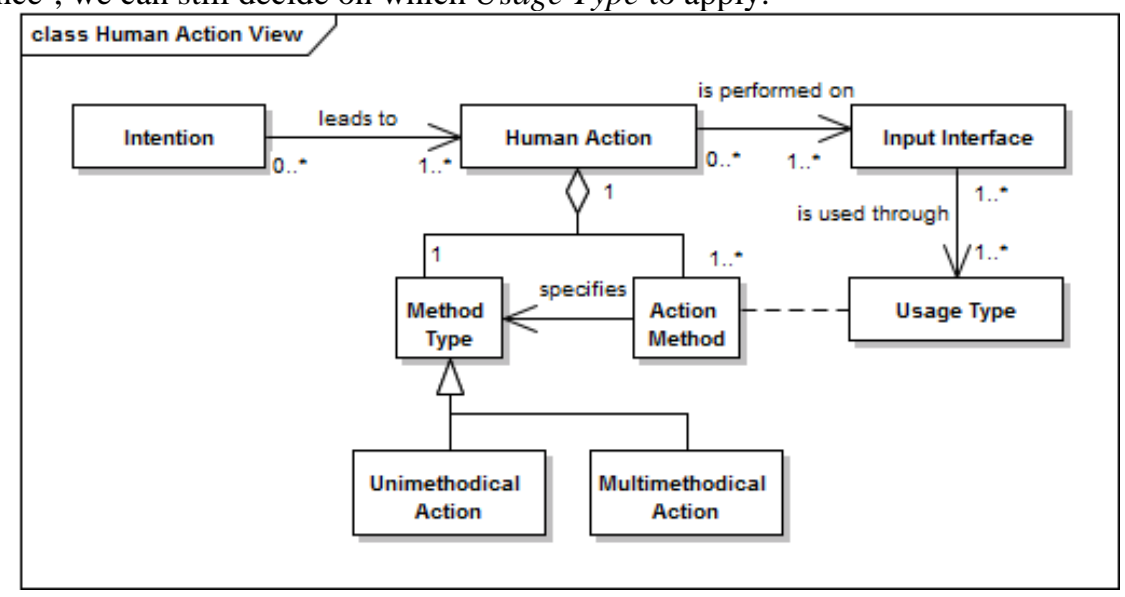

Fig. 3. Human Action View 


\subsection{System Action View}

The System Action view (see Figure 4) describes the way the System reacts to the Human Action and the transmission of information from the System to the Human.

The System Action is a composition of Direct Input Feedback, Indirect Input Feedback, Application Feedback, and System Function. Direct Input Feedback is feedback the Human gets directly from the Input Interface, i.e., not via any Medium. Example: the physical resistance of a keyboard stroke or the sound when pressing a key on a keyboard or a PC mouse. In contrast to this, Indirect Input Feedback is input feedback the Human gets via a Medium as a reaction to his usage of an Input Interface. It is the System Action on a human's action to confirm the system's correct understanding of that action. Indirect Input Feedback is always based on a System Function and can be influenced by a designer. Examples: bordering of an icon that the mouse cursor points to or highlighting of a selected menu entry. A System Function is a System Action that is performed automatically by the System as a reaction to an external trigger. An external trigger may be, for example, a Human Action, a call from an external system, or an environmental context change. A System Function does not necessarily address a Human directly, but recognizes events, interprets and manipulates data, and plans and initiates Application Feedback and Indirect Input Feedback. A System Function cannot be influenced by an interaction designer. Examples: an internal change in the system state, working hard disk drives, control of external but system-related objects (e.g., lights).

From an interaction-centered point of view, a System Action is given when at least one Application Feedback exists. Furthermore, an arbitrary number of the other elements can be included. The rationale for this composition is that Application Feedback directly refers to the human's Intention. Without feedback to the Intention, we are not able to fulfill an Elementary Interaction. For example, if a user performs an action on a user interface and only receives the feedback that his input was successful, we do not refer to this as an Elementary Interaction. The user always has to perceive the part of the System Action that belongs to his Intention in order to complete an Elementary Interaction. This part is the Application Feedback.

Depending on the Environmental Context in which the System Action takes place, the System Action might be influenced by that context. For example, the System Action might be to transmit information to the Human by means other than sound when the environment is noisy, or to show relevant information to a particular person when it detects that that person is near.

The System Action triggers a human's Perception, which is composed according to the System Action. Because of this segmentation, we can trace which Perception is triggered by which kind of System Action.

For each kind of Perception, a Modality Type is specified that determines if the Perception is unimodal or multimodal. Unimodal perception is given when exactly one Modality is used to perceive the part of the System Action. In multimodal perception, at least two different Modalities are used. 
In addition to that, for Indirect Input Feedback Perception and Application Feedback Perception, a Medium Type is specified that determines if the Perception is unimedial or multimedial. This is necessary for those two types because they are the only ones that are transmitted via a Medium. If one Medium is used for transmission, the medium type is unimedial; if at least two different Media are used, the medium type is multimedial.

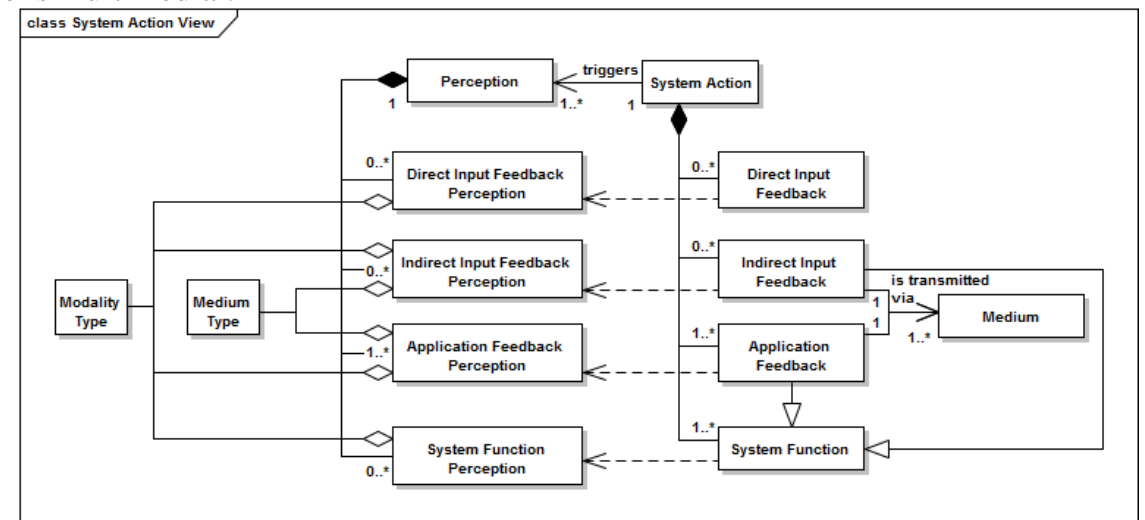

Fig. 4. System Action View

\subsection{Interaction View}

The interaction view (see Figure 5) gives a holistic overview of the views described so far and emphasizes the build-up of an Interaction. An Interaction consists of several Elementary Interactions. Furthermore, each Interaction consists of a $\mathrm{Hu}$ man Action that is initiated by an Intention and a System Action that triggers a Perception. This Perception either confirms or rejects the initial Intention. With the help of the interaction view, it is possible to describe an Interaction as a whole in a detailed manner, which can further be broken down by using more detailed views.

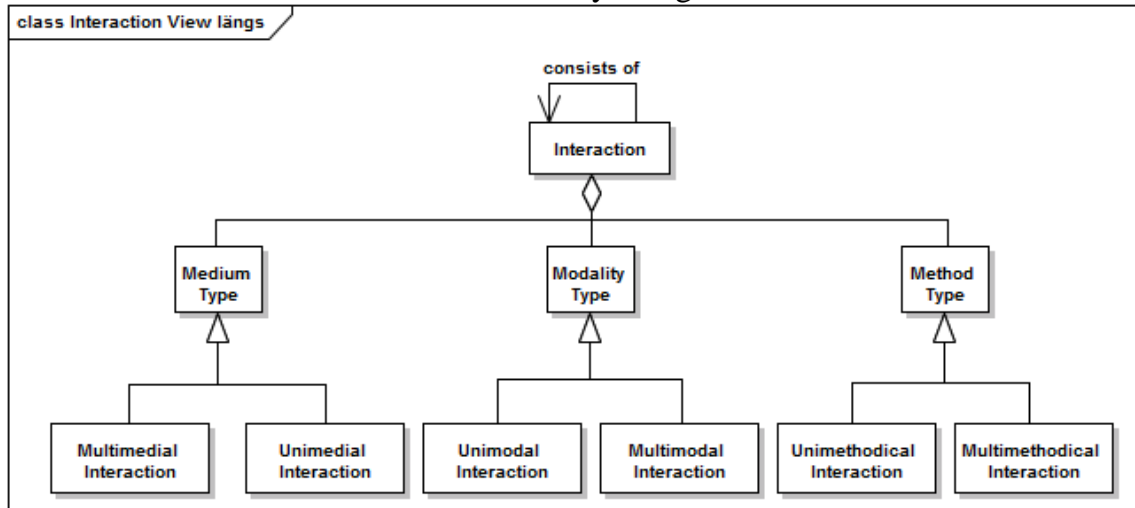

Fig. 5. Interaction View 


\subsection{Elementary Interaction View}

The Elementary interaction view (see Figure 6) describes the composition of an $\mathrm{El}$ ementary Interaction and its specializations in detail. Furthermore, it is shown how an Elementary Interaction is constructed.

Elementary Interactions are restricted to exactly one Human Action and one to many System Actions. Example: Typing text into a word processing application $\mathrm{(Hu}-$ man Action) and perceiving the written text on a screen in order to check the correct spelling (text output as System Action).

An Elementary Interaction always has three types, which specify how the Elementary Interaction takes place in detail.

First, the Method Type specifies if the Elementary Interaction is unimethodical or multimethodical. A unimethodical Elementary Interaction is an Elementary Interaction where the Human Action is unimethodical. A simple example is pressing a key on the keyboard in order to select a list item and visually perceiving the selected item. The attribute that is relevant for determining the Method Type derives directly from the Human Action; i.e., a unimethodical action remains unimethodical on the Elementary Interaction level, a multimethodical action remains multimethodical. Of course, in this simple example we have just one Action Method, namely pressing a key. A multimethodical Elementary Interaction is an Elementary Interaction where the $\mathrm{Hu}$ man Action is multimethodical. A simple example is pointing with a finger to an icon and saying 'open that' and visually perceiving the opening application. In this example, two different action methods are used in parallel during the action, namely 'Gesturing' and 'Speaking'. Therefore, the Elementary Interaction is multimethodical.

Second, the Modality Type specifies if the Elementary Interaction is unimodal or multimodal. A unimodal Elementary Interaction is an Elementary Interaction where Perception is unimodal. An example is requesting information from a speech dialog system on the phone and aurally perceiving the answers. A multimodal Elementary Interaction is an Elementary Interaction, where the Perception is multimodal. An example is requesting information from a speech dialog system on the PC and aurally as well as visually perceiving the answers.

To determine the modality types, all Perceptions are subsumed and it does not matter which kind of Perception the modality derives from.

Third, the Medium Type specifies if the Elementary Interaction is unimedial or multimedial. A unimedial Elementary Interaction is an Elementary Interaction where the Perception is unimedial. An example is using a command-based input shell (as in UNIX), perceiving only alphanumerical text. A multimedial Elementary Interaction is an Elementary Interaction where the Perception is multimedial. An example is using a GUI-based operating system (like Microsoft Windows) and perceiving alphanumerical text, graphics, animations, and icons during one single elementary interaction. To determine the Medium Type, each medium involved in Indirect Input Feedback Perception and Application Feedback Perception is considered.

An Elementary Interaction always has to be characterized through the triple $<$ method type, modality type, medium type >. For example, the Elementary Interaction of clicking the recycle bin icon on a MS Windows desktop to open the context menu 
is multimethodical (moving an object and pressing an object), unimodal (visual perception of the moving mouse cursor and the opening context menu), and multimedial (representation of information via the icon, text, and graphics within the context menu). An Elementary Interaction specified by only one or two of these types does not exist from our point of view.

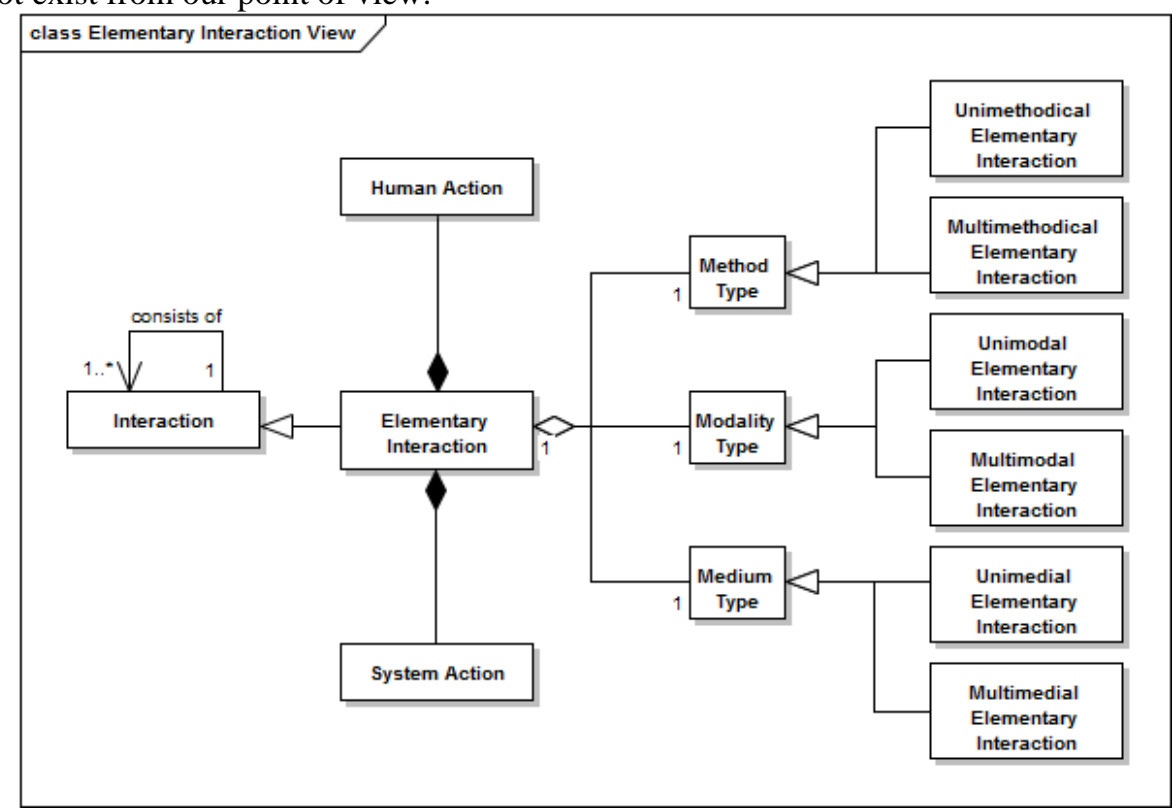

Fig. 6. Elementary Interaction View

\section{A Non-Formal Method Based on MAInEEAC}

When collaborating with non-expert stakeholders in order to define an interaction concept for a particular software system, MAInEEAC as a formal model is not applicable in its original condition.

Therefore, we have developed non-formal methods based on this formal model to facilitate this collaboration. After having accomplished a huge number of software engineering projects with interaction design activities, we have found that these methods, where stakeholders can speak freely, lead to more relevant information than strict formal and restricted approaches. One of these methods is the mConcAppt method [2],[3], which is a method for the user-centered conception of mobile business apps. In this chapter, we focus on the part of mConcAppt that is relevant for the construction of the HCI.

\subsection{Upfront Requirements Elicitation and Analysis}

In this practical approach for mobile interaction design, we face typical challenges like short time to market, high user experience, and less focused user attention than 
with desktop systems. Furthermore, the user interface design is related to device, platform, and technology. It comprises upfront requirements elicitation and analysis in combination with an iterative interaction design (see Figure 7). Both phases are conducted with close user involvement.

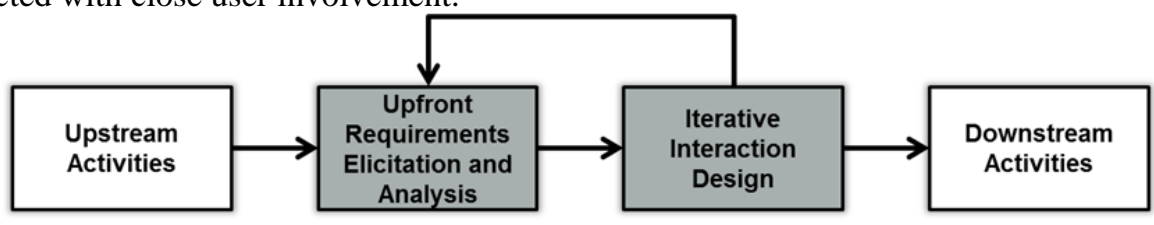

Fig. 7. Brief overview of mConcAppt

During the execution of mConcAppt, different kinds of artifacts are produced. Upfront requirements elicitation and analysis are mainly performed by means of a requirements elicitation workshop that involves end users as well as other projectrelevant stakeholders (see Figure 8). In addition, several requirements elicitation phone calls or interviews might be performed in order to clarify information elicited in the workshop as well as to elicit further requirements. As a result from upfront requirements elicitation and analysis, the following artifacts are produced in close collaboration with non-expert stakeholders:

1. List of involved stakeholders

2. Stakeholder goals

3. Stakeholder description

4. Description of a user persona [1]

5. Description of the as-is situation

6. Problems in the as-is situation

7. Description of the to-be situation

8. Technical constraints

9. Exchanged domain data

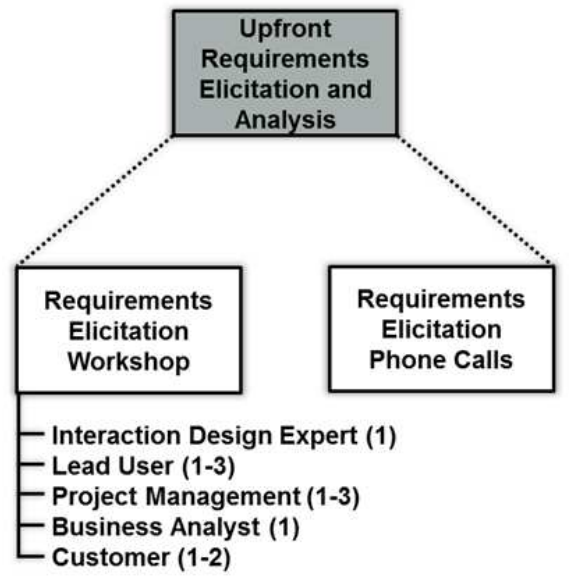

Fig. 8. Upfront Requirements Elicitation and Analysis Phase 
Requirements and interaction design experts already have the formal MAInEEAC model in mind while eliciting and documenting those artifacts. From the list of involved stakeholders, the definite end users (cf. Human in MAInEEAC) are derived. Especially stakeholder goals elicited in combination with the description of the to-be situation will ultimately lead to the interaction created using the formal model. Already at this point in time, communication between the requirements engineer and the interaction designer is improved, since they both have a conjoint formal model in mind when discussing different aspects.

All mentioned artifacts are used as input for the development of the initial interaction design, which will be described in the following chapter.

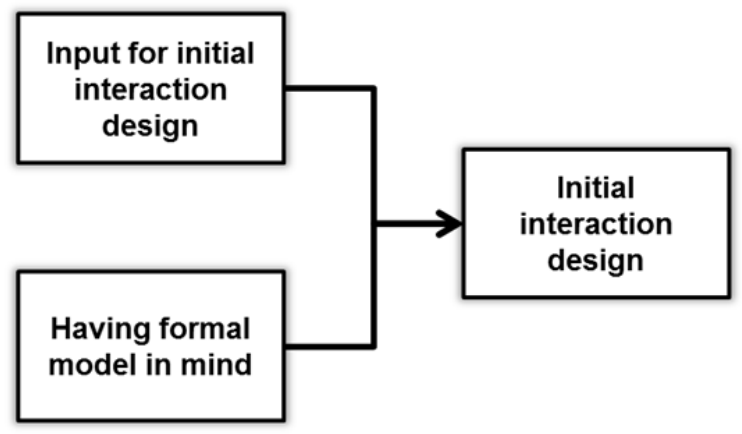

Fig. 9. Initial Interaction Design Phase

\subsection{Iterative Interaction Design}

The second phase during mConcAppt is the actual interaction design phase. Figure 9 shows that the artifacts elicited in the first phase and the formal model that the interaction designer has in mind are combined to create the interaction design. Therefore, interaction cases (see the example in Table 1Table 1) are created that structure the elicited to-be situation into a textual description using elements of the MAInEEAC model. During the textual specification of Interaction Cases, Action Methods, Usage Types, Input Feedback, and Application Feedback are defined. Using the interaction cases, the actual wireframes are assembled by combining exactly one Human Action and one System Action into one wireframe. In early iterations, wireframes are usually produced using paper prototypes and evaluated with real end users in a Wizard-of $\mathrm{Oz}$ setting. During those early evaluations and especially during later ones, when the prototype is already in a more mature state, the interaction designer benefits from having the formal model in mind when talking to the end user in order to check whether all recommended elements (e.g., giving Input Feedback at each Human Action) have been considered. In this phase, it might also beneficial to apply a co-design practice: interaction designers and end-users might create the interaction cases and wireframes conjointly; this will probably minimize the effort necessary for reworks of the interaction design after it was evaluated by other end-users. 


\begin{tabular}{|l|l|}
\hline Item & Description \\
\hline ID & IC2: Track time \\
\hline Usage Context & $\begin{array}{l}\text { Business travel for multiple days. Meeting an industrial } \\
\text { partner in Leipzig. The user is on his way to the hotel and } \\
\text { uses the device while walking. }\end{array}$ \\
\hline System Action 1 & $\begin{array}{l}\text { The system recognizes that the user arrives at his destina- } \\
\text { tion and notifies him that the time of arrival is tracked } \\
\text { (via the notification center). }\end{array}$ \\
\hline Human Action 1 & The user taps the notification to directly open the app. \\
\hline System Action 2 & $\begin{array}{l}\text { The system opens the app and immediately shows the } \\
\text { current trip itinerary and the proposed time. }\end{array}$ \\
\hline Human Action 2 & The user confirms the proposed time. \\
\hline System Action 3 & $\begin{array}{l}\text { The system provides feedback on the confirmation to the } \\
\text { user. }\end{array}$ \\
\hline Human Action 3 & The user closes the app. \\
\hline Post-condition & The arrival time is stored persistently. \\
\hline
\end{tabular}

Table 1. Interaction Case Example

\section{$4 \quad$ Advantages of Our Approach}

The application of the approach we propose in this chapter bears a number of advantages: Non-expert stakeholders are not forced to worry about formal models, since expert stakeholders do not discuss formal models with non-expert stakeholders, but rather prepare their conversations with the help of the model. Due to the unrestricted and non-formal conversations, all stakeholders can speak freely and more relevant information is gathered than with strict formal and restricted approaches like workshops and interviews. During the conversation, the expert stakeholders know which information is missing and can describe and discuss the impact of decisions made collaboratively. But it is still the interaction designer who creates the final interaction design and the developer who implements the interaction. Benefits of our approach can also be found within the groups of expert stakeholders and non-expert stakeholders, where communications are facilitated, since a single model with a single terminology can be used. When single group members are familiar with or used to another terminology, both terminologies can be mapped very easily. Besides requirements engineers, interaction designers, and developers, visual designers, architects, business analysts, customers, and end-users will also benefit from this facilitation of communication. The requirements elicited, analyzed, and specified by these communications while applying the non-formal method can be traced to concrete interactions and their 
highly detailed elements. While the formal model represents the elements, the nonformal method assures traceability.

\section{Conclusion}

From our point of view, early collaboration with non-expert stakeholders is mandatory. This is done best by applying non-formal methods based on formal models. Insisting on formal modeling when collaborating with non-expert stakeholders leads to insufficient results, since non-expert stakeholders have to familiarize themselves with unusual formal models and unusual formal thinking instead of focusing on considerations about needs, wishes, and demands in terms of HCI.

In this article, we presented our user-centered design approach for an interaction design based on our non-formal method mConcAppt, which follows our formal model MAInEEAC. Due to the early involvement of non-expert stakeholders, the software development process can be shortened and thus could be applied to a wide range of software development projects, especially to projects using an agile development approach. Such agile development approaches are often applied to mobile business applications, for example, which need a lightweight user-centered approach because of their special challenges. Formal approaches do not satisfy this requirement sufficiently. The approach can be applied to a large number of domains due to its flexibility and the formal structure in the background.

The approach presented (MAInEEAC, mConcAppt and their interrelation) is based on best practices resulting from many projects in which we have designed interaction in collaboration with non-expert stakeholders, and is even supposed to enable semiexperts to design a well-conceived HCI. An example of the application of the approach in an actual project can be found in [5]. However, the approach is still evolving and work is in progress. We plan to integrate interaction-related aspects such as user experience and architecture in order to be able to cover a holistic view on HCI and to discuss all relevant aspects with non-expert stakeholders. We also believe that this approach is able to create new value through its capability to apply the cocreation practice. However, investigations of such effects by applying the approach were not conducted yet and remain an open issue to carry out as future work. Eventually, we hope to achieve a huge increase in interaction design quality with the help of our approach.

\section{References}

1. Cooper, A.: The Inmates Are Running the Asylum: Why High-Tech Products Drive Us Crazy and How to Restore the Sanity. Indianapolis, USA, 1999.

2. Hess, S., Kiefer, F.: mConcAppt Methode - UX und Interaktionsdesign für mobile Business Apps in Usability Professionals Association, German Chapter: Usability Professionals 2012 Tagungsband. Konstanz, 2012.

3. Hess, S., Kiefer, F.: Quality by Construction through mConcAppt - Toward Using UIConstruction as a Driver for High Quality Mobile App Engineering. QUATIC 2012. 
4. Hess, S., Maier, A., Trapp, M.: Differentiating between Successful and Less Successful Products by Using MAInEEAC - A Model for Interaction Characterization in HumanComputer Interaction. Design and Development Approaches, Springer Berlin / Heidelberg, 2011.

5. Hess, Steffen ; Kiefer, Felix ; Carbon, Ralf ; Maier, Andreas: mConcAppt - A Method for the Conception of Mobile Business Applications. In: Uhler, David (Ed.) ; Mehta, Khanjan (Ed.) ; Wong, Jennifer L. (Ed.): Mobile Computing, Applications, and Services. 4th International Conference. MobiCASE 2012 - Revised Selected Papers. Berlin: Springer-Verlag, 2013, 1-20. (Lecture Notes of the Institute for Computer Sciences, Social Informatics and Telecommunications Engineering 110).

6. International Organization for Standardization. ISO 9241-210:2010 - Ergonomics of human-system interaction -- Part 210: Human-centred design for interactive systems, 2010.

7. Norman, D. A.: Some observations on Mental Models. In D. GENTNER, and A.L. STEVENS, eds. Mental Models. Lawrence Erlbaum Associates Inc. pp7-14, 1983.

8. Norman, D. A.: The Design of Everyday Things. New York, Doubleday, 1988.

9. Schomaker, L., Munch, S., Hartung, K.: A taxonomy of multimodal interaction in the human information processing system. Technical report, ESPRIT BRA, No. 8579, 1995. 